\title{
Density Measurement for the Binary Mixtures of Ionic Liquid 1- ethyl- 3-methylimidazolium Diethylphosphate and Water (Methanol or Ethanol) at $1 \mathrm{~atm}$ and (293.15 to 333.15$) \mathrm{K}$
}

\author{
Yin-Hui Gong ${ }^{\mathrm{a}}$, Ying-Zhou Lü ${ }^{\mathrm{b}}$, Hong Meng ${ }^{\mathrm{b}}$ and Chun-Xi Li $\mathrm{Li}^{\mathrm{a}, \mathrm{b}, *}$ \\ ${ }^{a}$ State Key Laboratory of Chemical Resource Engineering; ${ }^{b}$ College of Chemical Engineering, Beijing \\ University of Chemical Technology, Beijing 100029, P.R., China
}

\begin{abstract}
The density data for the binary mixtures of an ionic liquid (IL), 1-ethyl-3-methylimidazolium diethylphosphate [EMIM][DEP], and water (methanol or ethanol) were measured at $1 \mathrm{~atm}$ as a function of composition in the temperature range of (293.15 to 333.15$) \mathrm{K}$ using a vibrating-tube densimeter. The excess molar volumes $\left(V^{E}\right)$ and other thermodynamic properties were derived from the density data. All $V^{E}$ values are negative for the binary mixtures in the whole composition range, and reach to the maximum at the mole fraction of IL of ca. 0.3. The $V^{E}$ values decrease with increasing temperature for the aqueous solution of ILs, but increase with the increasing temperature for the IL solutions of methanol or ethanol. The excess molar volumes were correlated successfully by an empirical equation with the maximum average absolute relative deviation within $0.02 \%$.
\end{abstract}

Keywords: Density, excess volume, ionic liquid, water, methanol, ethanol.

\section{INTRODUCTION}

Ionic liquids (ILs), as a liquid electrolyte at ambient temperatures, have been attracting the attention of a growing number of scientists and engineers, because of their unique properties, such as negligible volatility and good conductivity. ILs may be used as alternative solvents for many separation and reaction processes, for instance, as extractants for extractive distillation and extraction process in chemical industry [1, 2], or as solvents for chemical reactions [3]. To design taskspecific ILs and the industrial process for the ILcontaining systems, it is necessary to know the physical and thermodynamic properties of ILs and their solutions at varying conditions. Density is a fundamental property, which reflects the intermolecular interactions and the solution non-ideality from one aspect. As a continuation of our study on the thermodynamic properties of ILs [4-6], we measured the density of three binary mixtures containing water (methanol or ethanol) and [EMIM][DEP] at $1 \mathrm{~atm}$ in the temperature range of (293.15 to 333.15$) \mathrm{K}$. The excess molar volumes $\left(V^{E}\right)$ are calculated from the density data, and correlated as a function of temperature and composition by an empirical equation. The solution behavior of the IL-containing mixtures was discussed in terms of the $V^{E}$ variation with temperature and composition of the mixtures. By now, no literature data have been available for the densities and excess molar volumes of the mixtures studied herein.

*Address correspondence to this author at the State Key Laboratory of Chemical Resource Engineering, College of Chemical Engineering, Beijing University of Chemical Technology, Beijing 100029, P.R., China;

Tel/Fax: 86-10-64410308; E-mail: Licx@mail.buct.edu.cn

\section{EXPERIMENTAL}

\subsection{Materials}

The chemicals used for synthesizing [EMIM][DEP] are 1-methylimidazole ( $\geq 99.5 \mathrm{wt} . \%$, Zhejiang Kaile Reagents Company, China), triethylphosphate ( $\geq 99.5$ wt.\%, Tianjin Guangfu Reagents Company, China) and aether ( $\geq 99.5$ wt.\%, Beijing Yili Reagents Company, China), which were used as received. Methanol and ethanol were supplied by Beijing Chemical Plant with nominal purity above 99.7 wt.\%, dried by the activated molecular sieves of type $4 \AA$ (Union Carbide), and stored in tightly sealed glass bottle. [EMIM][DEP] was synthesized and purified in the laboratory according to the method described elsewhere [7], and its purity is above 99 wt.\% as determined by the NMR analysis. Water content in the ILs is below $0.032 \%$ as determined by the Karl Fisher titrator (type CBS-1A).

\subsection{Apparatus and Procedure}

The densities of pure ILs and their binary mixtures with water (methanol or ethanol) at different temperatures were measured using the digital vibrating tube densimeter (DMA 4500M, Anton Paar Co. Ltd., Austria). The binary mixtures with different mole fractions of ILs were prepared by weighting with an electronic balance (type AR 2130, Ohaus Co. Ltd., USA) with a weighing precision of $\pm 0.001 \mathrm{~g}$. The nominal uncertainty of the experimental density is \pm $1 \times 10^{-5} \mathrm{~g} \cdot \mathrm{cm}^{-3}$. The temperature of the densimeter measuring cell is better than $0.002 \mathrm{~K}$ and the precision of measurement is $\pm 0.01 \mathrm{~K}$ with a built-in thermostat. The accuracy of the density data is verified by 
Table 1: Density $\rho /\left(\mathrm{g} \cdot \mathrm{cm}^{-3}\right)$ of Pure ILs [EMIM][DEP] and Water at $1 \mathrm{~atm}$ and Varying Temperatures

\begin{tabular}{|c|c|c|c|c|c|c|c|c|c|}
\hline $\mathrm{T} / \mathrm{K}$ & 293.15 & 298.15 & 303.15 & 308.15 & 313.15 & 318.15 & 323.15 & 328.15 & 333.15 \\
\hline $\mathrm{IL}$ & 1.15221 & 1.14886 & 1.14550 & 1.14213 & 1.13876 & 1.13540 & 1.13204 & 1.12869 & 1.12534 \\
\hline water & 0.99820 & 0.99705 & 0.99565 & 0.99404 & 0.99224 & 0.99023 & 0.98805 & 0.98570 & 0.98306 \\
\hline
\end{tabular}

comparing the experimental data of water with the reference values [8] from 293.15 to 333.15 with the average relative deviation within $0.02 \%$.

\section{RESULTS AND DISCUSSION}

\subsection{Density and Derived Properties of [EMIM][DEP]}

The densities of pure ILs [EMIM][DEP] at 1 atm were measured in the temperature range of $(293.15$ to 333.15) $\mathrm{K}$, and listed in Table 1. It is found that the density of ILs is generally higher than that of molecular solvents due to the strong electrostatic interaction among ions of the ILs [9], and the density of the pure components follows the order ethanol $\approx$ methanol $<$ water $<[E M I M][D E P]$ in the same other conditions.

Density of IL [EMIM][DEP] was used to derive other thermodynamic properties such as the thermal expansion coefficient, molar volume, standard entropy and lattice energy. Thermal expansion coefficient $(\alpha)$ of a liquid is defined as follows:

$\alpha=\frac{1}{V}\left(\frac{\partial V}{\partial T}\right)_{\mathrm{P}}=-\left(\frac{\partial \ln \rho}{\partial T}\right)_{\mathrm{P}}$

where $V$ and $\rho$ is the molar volume and density of the IL, respectively. The variation trend of In $\rho$ versus $T$ for [EMIM][DEP] is presented in Figure 1. As shown in the figure, the values of In $\rho$ decrease slightly in a linear

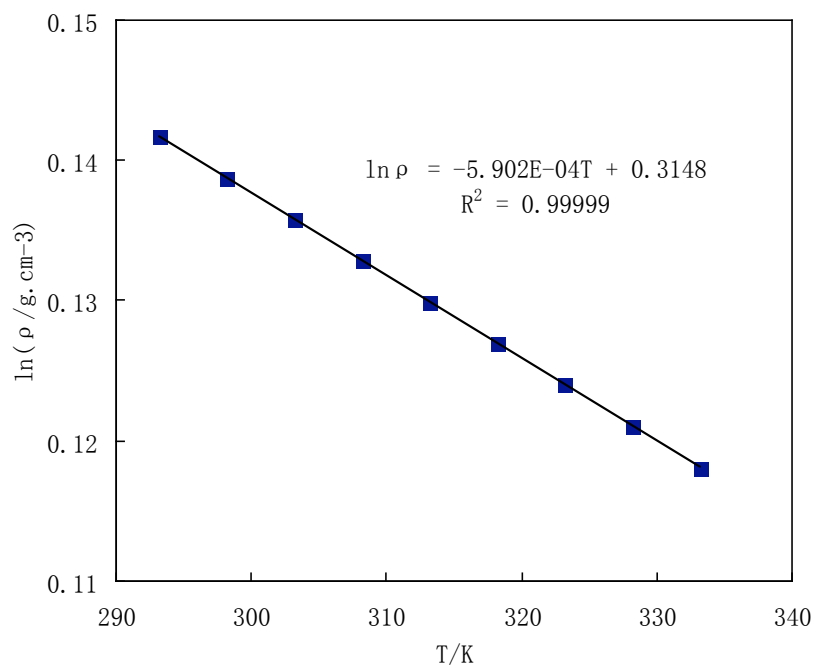

Figure 1: Plot of $\ln \left(\rho / \mathrm{g} \cdot \mathrm{cm}^{-3}\right)$ against $T / K$ for IL [EMIM][DEP]. way with the increasing temperature. The $\alpha$ value of [EMIM][DEP] is obtained from the slope of the linear regression of $\ln \rho$ against $T$ as $5.9 \times 10^{-4} \mathrm{~K}^{-1}$. The thermal expansion coefficient of [EMIM][DEP] is quite low, indicating IL is less compressible.

Molecular volume of the ILs, $V_{\mathrm{m}}$, can be estimated from the density data by Eqn (2):

$V_{\mathrm{m}}=M /\left(N_{\mathrm{A}} \cdot \rho\right)$

where $M$ is the molar mass, $N_{\mathrm{A}}$ is the Avogadro constant, $\rho$ is the density. The $V_{\mathrm{m}}$ value of [EMIM][DEP] at $298.15 \mathrm{~K}$ is calculated to be 0.3821 .

The standard entropy, $S^{\circ}$, is calculated in terms of the molecular volume and a good linear relationship proposed by Glasser [10]. The linear correlation constants in Eq. (3) are appropriate for the ILs with large organic cations.

$S^{0}(298) /\left(\mathrm{J} \cdot \mathrm{mol}^{-1} \cdot \mathrm{K}^{-1}\right)=1246.5\left(V_{\mathrm{m}} / \mathrm{nm}^{3}\right)+29.5$

The $S^{0}$ values calculated by Eq. (3) for the IL [EMIM][DEP] is $505.8 \mathrm{~J} \cdot \mathrm{mol}^{-1} \cdot \mathrm{K}^{-1}$.

Density data is also useful for the estimation of lattice energy (U $U_{\text {РОT }}$ ) of ILs. According to the Glasser's theory, the lattice energy depends only on the chemical formula, ionic charges and molecular volume of the materials involved, and neither relies on any other structural information. For the present case of ionic liquids of $M X$ type with charge ratio $1: 1$, the Columbic interaction is the principal contribution to the lattice energy, and the $U_{\text {POT }}$ values can be estimated by Eq. (4).

$U_{\mathrm{POT}} /\left(\mathrm{kJ} \cdot \mathrm{mol}^{-1}\right)=1981.2(\rho / M)^{1 / 3}+103.8$

The lattice energies of [EMIM][DEP] is 427.2 $\mathrm{kJ} \cdot \mathrm{mol}^{-1}$, which is obviously much lower than that of the fused inorganic salts, for example, the lattice energy of fused CsI which has the least crystal energy among all the alkali halides is about $602.5 \mathrm{~kJ} \cdot \mathrm{mol}^{-1}$ [11]. The low lattice energy may be the underlying reason for forming ILs at room temperature [12]. 
Table 2: Density Data Measured for the Binary Mixtures at 1 atm and Different Temperatures and Mole Fractions of ILs

\begin{tabular}{|c|c|c|c|c|c|c|c|c|c|}
\hline \multirow{2}{*}{$\mathbf{x}_{\mathrm{IL}}$} & \multicolumn{9}{|c|}{ Density $\rho /\left(\mathrm{g} \cdot \mathrm{cm}^{-3}\right)$ at the following temperatures, $\mathrm{T} / \mathrm{K}$} \\
\hline & 293.15 & 298.15 & 303.15 & 308.15 & 313.15 & 318.15 & 323.15 & 328.15 & 333.15 \\
\hline \multicolumn{10}{|c|}{ [EMIM][DEP] + water } \\
\hline 0.200 & 1.14272 & 1.13912 & 1.13545 & 1.13176 & 1.12804 & 1.12428 & 1.12051 & 1.11672 & 1.11287 \\
\hline 0.399 & 1.15274 & 1.14928 & 1.14580 & 1.14231 & 1.13881 & 1.13529 & 1.13176 & 1.12822 & 1.12466 \\
\hline 0.602 & 1.15389 & 1.15052 & 1.14713 & 1.14372 & 1.14031 & 1.13689 & 1.13347 & 1.13004 & 1.12661 \\
\hline 0.799 & 1.15319 & 1.14985 & 1.14648 & 1.14310 & 1.13972 & 1.13634 & 1.13296 & 1.12959 & 1.12622 \\
\hline \multicolumn{10}{|c|}{ [EMIM][DEP] + methanol } \\
\hline 0.199 & 1.01922 & 1.01542 & 1.01161 & 1.00779 & 1.00398 & 1.00016 & 0.99634 & 0.99252 & 0.9887 \\
\hline 0.400 & 1.08843 & 1.08485 & 1.08126 & 1.07767 & 1.07408 & 1.07051 & 1.06694 & 1.06336 & 1.05974 \\
\hline 0.601 & 1.12065 & 1.11716 & 1.11368 & 1.11021 & 1.10675 & 1.10329 & 1.09984 & 1.09639 & 1.09294 \\
\hline 0.800 & 1.13996 & 1.13659 & 1.13317 & 1.12976 & 1.12635 & 1.12295 & 1.11956 & 1.11618 & 1.11280 \\
\hline \multicolumn{10}{|c|}{ [EMIM][DEP] + ethanol } \\
\hline 0.201 & 0.97907 & 0.97530 & 0.97153 & 0.96776 & 0.96398 & 0.96020 & 0.95641 & 0.95262 & 0.94881 \\
\hline 0.398 & 1.05862 & 1.05506 & 1.05147 & 1.04788 & 1.04430 & 1.04072 & 1.03714 & 1.03355 & 1.02995 \\
\hline 0.600 & 1.10354 & 1.10005 & 1.09658 & 1.09312 & 1.08966 & 1.08620 & 1.08274 & 1.07929 & 1.07584 \\
\hline 0.799 & 1.13217 & 1.12879 & 1.12537 & 1.12196 & 1.11856 & 1.11517 & 1.11178 & 1.10839 & 1.10501 \\
\hline
\end{tabular}

\subsection{Density of the Binary Mixtures}

The density data for the IL-containing binary mixtures at the mole fraction of $\mathrm{IL}, x_{\mathrm{IL}}$, being about 0.2 , $0.4,0.6$, and 0.8 , were measured at the same pressure and temperature as that for the pure components, and listed in Table 2. The variation trend of density with temperature and IL-content for a binary mixture is presented in Figure 2. As shown in the figure, the densities of a mixture are always between that of the constituting pure components, i.e., $\rho_{\mathrm{IL}}>\rho_{\text {mixture }}>\rho_{\text {solvent, }}$

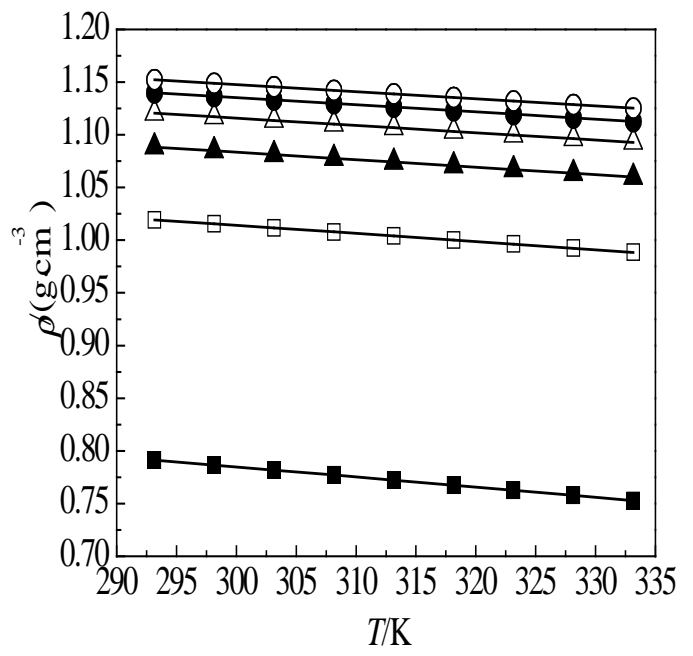

Figure 2: The experimental (symbols) and correlative (lines) density of [EMIM][DEP] + methanol binary mixtures at different temperatures and mole fractions of IL: $(\mathbf{\square}), 0 ;(\square)$, 0.199; ( $\Delta), 0.400 ;(\triangle), 0.601 ;(\bullet), 0.800 ;(O), 1$. and decrease slightly in a linear way with the increasing temperature and increase rapidly with the IL-content.

\subsection{Excess Molar Volume of the Binary Mixtures}

The excess molar volume, $V^{E}$, is an important thermodynamic property to represent the non-ideality of a solution, which is defined as the difference of the molar volume between the real mixture $\left(V_{m}\right)$ and the ideal solution $\left(\mathrm{V}_{\mathrm{m}, \mathrm{id}}\right)$ at the same temperature, pressure and composition i.e.

$$
\begin{aligned}
& V^{\mathrm{E}}=V_{\mathrm{m}}-V_{\mathrm{m}, \mathrm{id}} \\
& V_{\mathrm{m}} \text { and } V_{\mathrm{m}, \mathrm{dd}} \text { values are calculated as follows: } \\
& V_{\mathrm{m}}=\frac{x_{\mathrm{IL}} M_{\mathrm{IL}}+x_{\mathrm{S}} M_{\mathrm{S}}}{\rho_{\text {mix }}} \\
& V_{\mathrm{m}, \mathrm{d}}=\frac{x_{\mathrm{IL}} M_{\mathrm{IL}}}{\rho_{\mathrm{IL}}}+\frac{x_{\mathrm{S}} M_{\mathrm{S}}}{\rho_{\mathrm{S}}}
\end{aligned}
$$

where $M_{\mathrm{S}}$ and $M_{\mathrm{L}}$ are the mole masses of solvent and $\mathrm{IL}$, respectively. $x_{\mathrm{S}}$ and $x_{\mathrm{IL}}$ represent the mole fractions of solvent and IL, respectively. $\rho_{\mathrm{S}}, \rho_{\mathrm{IL}}$ and $\rho_{\text {mix }}$ refer to the densities of solvent, IL and the mixture, respectively.

Based on the density data, as listed in Tables 1 and 2 , the excess molar volume of the binary mixtures at 
different conditions were calculated using Eqs. 5 to 7. The results show that the excess volumes are negative for all the systems studied at any temperature and concentration, indicating a negative deviation from the ideal solution behavior. Besides, the excess volume is relatively low being in the range of $(0.9$ to 1.9$) \mathrm{cm}^{3} \cdot \mathrm{mol}^{-}$ ${ }^{1}$ for all the binary mixtures, and reaches the maximum at the mole fraction of $\mathrm{IL}$ of ca. 0.3. For the convenience of illustration, plots of excess molar volume against the mole fraction of $\mathrm{IL}$ at different temperatures for the binary mixtures of [EMIM][DEP] with methanol and water are presented in Figures $\mathbf{3}$ and 4, respectively. As shown in Figure 3, the excess molar volume of the ([EMIM][DEP] + methanol) mixture increases with increasing temperature at fixed other conditions. This trend holds for ([EMIM][DEP] + ethanol) mixture as well and in good agreement with those reported elsewhere for other ILs mixtures with different alcohols [13, 14].

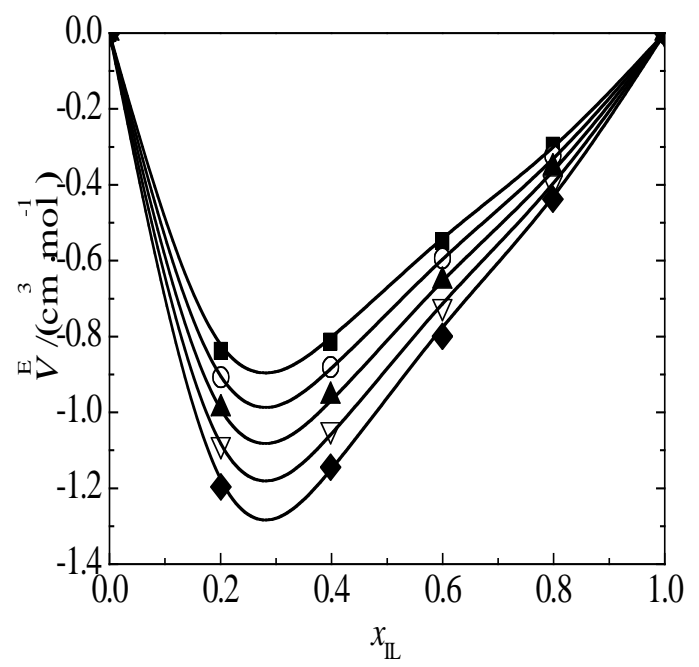

Figure 3: The experimental and correlative excess molar volume for the binary mixture ([EMIM][DEP] + methanol) at different mole fractions of IL and temperatures: (ם), 293.15 $\mathrm{K}$; ( O ), 303.15 K; ( $\Delta$ ), 313.15 K; $(\nabla), 323.15 \mathrm{~K} ;(\bullet), 333.15$ $\mathrm{K} ;(-)$, calculated values from Eq. (8).

In contrast to the binary mixtures of IL plus alcohols, the excess volumes of the (IL + water) mixtures decrease as the temperature increasing, as shown in Figure 4, implying that the non-ideality of the mixture is higher at lower temperature and decreases as the temperature increasing. This variation trend may be attributed to the strong dielectric property of water and its decreasing dielectric behavior as temperature increasing, for example, the dielectric constant of water decreases from 80.1 at $293 \mathrm{~K}$ to 63.8 at $333 \mathrm{~K}$ [8]. The high dielectric property of water makes the ionic liquid partially dissociated into ions, leading to a high asymmetrical interaction between neutral water molecules and charged particles of the IL component, and accordingly a high non-ideality of the mixture and the excess volumes. This means that the aqueous solution of ILs usually shows a larger deviation from the ideal solution behavior than an alcoholic solution of ILs, which is justified by the fact that the values of $V^{E}$ for the (IL + water) mixtures are always larger than those for the $(\mathrm{IL}+$ alcohol) mixtures at the same temperature and concentration. Besides, as the temperature increases the ILs component is apt to present as neutral molecules rather than as ionic species due to the decreasing dielectric constant of water, leading to a lower non-ideality and excess volume.

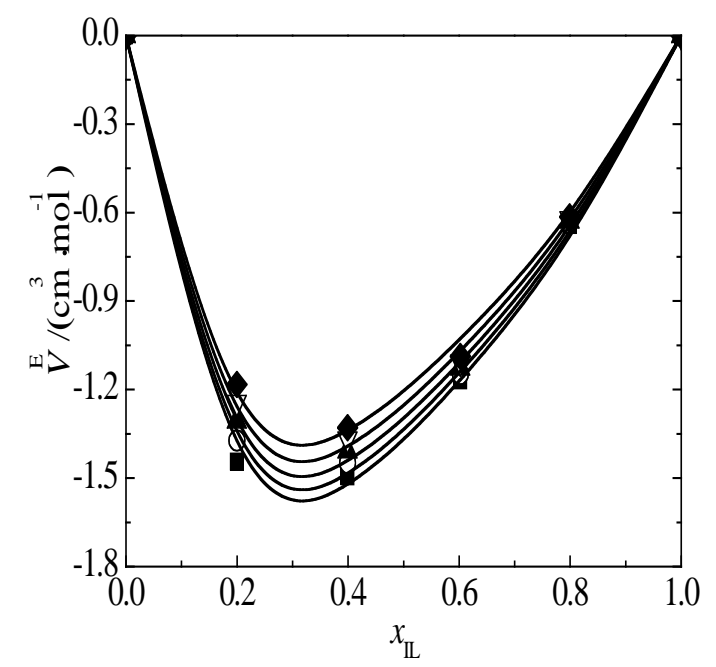

Figure 4: The experimental and correlative excess molar volume for the binary mixture ([EMIM][DEP] + water) at different mole fractions of IL and temperatures: $(\boldsymbol{\square}), 293.15$ $\mathrm{K} ;(\mathrm{O}), 303.15 \mathrm{~K} ;(\boldsymbol{\Delta}), 313.15 \mathrm{~K} ;(\nabla), 323.15 \mathrm{~K} ;(\bullet), 333.15$ $\mathrm{K} ;(-)$, calculated values from Eq. (8).

For a specific ionic liquid, the solvent property has an important influence on the nonideality and excess molar volume of the IL-containing binary solutions. Figure 5 presents the excess molar volumes against the mole fraction of $\mathrm{IL}$ for the binary mixtures of [EMIM][DEP] with water, methanol and ethanol at $298.15 \mathrm{~K}$. It is seen that among the three binary mixtures, the aqueous solution shows the highest excess molar volume and the ethanol solution shows the lowest one at the same temperature and mole fraction of $\mathrm{IL}$. This trend is in agreement with the dielectric constant [8] order at $298.15 \mathrm{~K}$, viz. water (78.3) > methanol (32.7) > ethanol (24.5). As a result, the aqueous solution of IL is more like an electrolyte solution, while the alcoholic solution of IL is more like a non-electrolytic organic mixture. In effect, the excess volume is a reflection of the compromising effects 
among molecular sizes and various interactions for a specific mixture, and is dependent on the temperature and composition. The negative excess volume may be associated with the strong ion-dipole and dipole-dipole interactions between $\mathrm{IL}$ and the solvent molecules, as well as the interstitial effect in molecular packing due to the differences in size and shape of the component molecules, by which the volume of the liquid mixture is apt to be contracted.

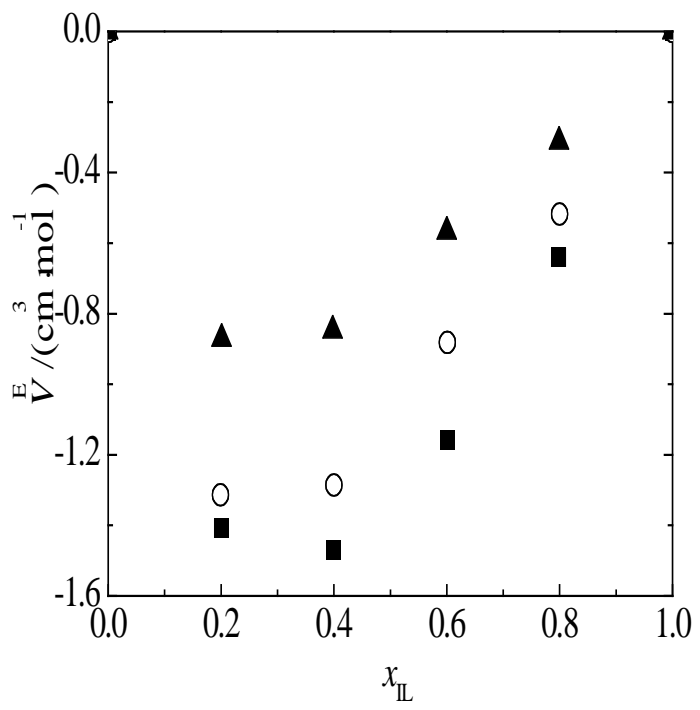

Figure 5: Excess molar volume for the binary mixtures of [EMIM][DEP] with different solvents at 298.15K: (ם), $[$ EMIM][DEP] + water; (O), [EMIM][DEP] + methanol; (4), $[E M I M][D E P]+$ ethanol.

\subsection{Correlation of the Density Data}

In view of the complex variation of the excess molar volume with temperature and IL-content for a binary mixture, the $V^{E}$ values are fitted to equation (8) by the least-square method.
$V^{\mathrm{E}}=x_{\mathrm{IL}}\left(1-x_{\mathrm{IL}}\right) \sum_{\mathrm{i}=0}^{\mathrm{j}} A_{\mathrm{i}}\left(1-2 x_{\mathrm{IL}}\right)^{\mathrm{i}}\left(1+B T+C T^{2}\right)$

Where $x_{\mathrm{IL}}$ is the mole fraction of $\mathrm{IL}, T$ is the temperature in $\mathrm{K}$. It was found that the best fit was obtained when five adjustable parameters were used in Eq. 8. The resulting parameters $A_{0}, A_{1}, A_{2}, B, C$, and the correlation deviations in average absolute relative deviation, i.e. $\operatorname{AARD}\left(V^{E}\right)$, or mean absolute percent deviation (MAPD) as noted by McHugh et al. [15,16], for each binary system are listed in Table 3 . The experimental density data were accurately correlated by Eqs (5) through (8) with the maximum correlative deviation of AARD $(\rho)$ within $0.02 \%$, which is close to the experimental uncertainty of the densimeter used here. The goodness of Eqs (5) through (8) for representing density and excess molar volume of the IL-containing binary mixtures can be clearly seen from Figures 2 through $\mathbf{4}$, as well as the very low AARD values as listed in Table $\mathbf{3}$ and Figure $\mathbf{6}$.

\section{CONCLUSIONS}

The density data for the binary mixtures of [EMIM][DEP] and a solvent of water(methanol or ethanol), were measured at $1 \mathrm{~atm}$ in the temperature range of (293.15 to 333.15) K using the densimeter. The excess volumes are negative in the whole temperature and concentration range studied, indicating a negative deviation from the ideality, and the variation of excess volume with temperature shows two opposite trends for the (IL + water) mixtures and the (IL + alcohol) mixtures. Moreover, the excess volume as well as the density data can be correlated accurately by a five-parameter empirical polynomial equation.

Table 3: The Regressed Parameters of Eq. (8) and the Deviation in AARD for the Binary Systems

\begin{tabular}{|c|c|c|c|}
\hline $\begin{array}{c}\text { Correlation results } \\
\text { [EMIM][DEP]IL }\end{array}$ & IL + water & IL + methanol & IL + ethanol \\
\hline \hline$A_{0}$ & -0.00187 & -0.00031 & -0.00042 \\
\hline$A_{1}$ & -0.00123 & -0.00030 & -0.00042 \\
\hline$A_{2}$ & -0.00085 & -0.00027 & -0.00034 \\
\hline$B$ & 26.63691 & 4.15221 & -20.16439 \\
\hline$C$ & -0.05662 & 0.14370 & 0.14385 \\
\hline $\operatorname{AARD}\left(V^{E}\right)$ & $1.5385 \%$ & $0.3359 \%$ & $0.7035 \%$ \\
\hline $\operatorname{AARD}(\rho)$ & $0.0167 \%$ & $0.0031 \%$ & $0.0037 \%$ \\
\hline
\end{tabular}

$\mathrm{AARD}=\frac{1}{n} \sum_{\mathrm{i}=1}^{n}\left|V^{\mathrm{E}(\text { cal })} / V^{\mathrm{E}(\text { exp })}-1.0\right|$ 


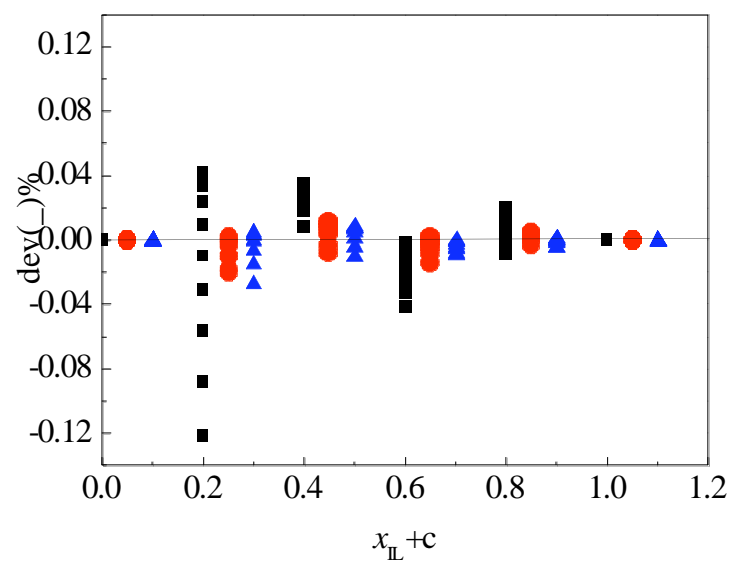

Figure 6: Relative deviations of density correlation in the whole concentration range and temperature range of (298.15 333.15K) for the binary mixtures. (ם), [EMIM][DEP] + water $(\mathrm{C}=0) ;(\bullet)$, [EMIM][DEP] + ethanol $(\mathrm{C}=0.05) ;(\boldsymbol{\Delta})$, $[$ EMIM] $[D E P]+$ methanol $(\mathrm{C}=0.1)$.

\section{ACKNOWLEDGEMENTS}

The authors are grateful to the financial support from National Natural Science Foundation of China (21076005) and Research Fund for the Doctoral Program of Higher Education of China (20090010110001).

\section{REFERENCES}

[1] Seiler M. Jork C. Kavarnou A. Arlt W. Hirsch R. Separation of azeotropic mixtures using hyperbranched polymers or ionic liquids. AIChE J 2004; 50: 2439-54. http://dx.doi.org/10.1002/aic.10249

[2] Wang R. Wang J. Meng H. Li C. Wang Z. Liquid-Liquid equilibria for benzene /cyclohexane mixture containing ionic liquid 1-methyl-3-methylimidazolium dimethyl phosphate or 1-ethyl-3-methylimidazolium diethyl phosphate. J Chem Eng Data 2008; 53: 1159-62. http://dx.doi.org/10.1021/je700759h

[3] Zhao D. Wu M. Kou Y. Min E. Ionic liquids: applications in catalysis. Catalysis Today 2002; 74: 157-89. http://dx.doi.org/10.1016/S0920-5861(01)00541-7

[4] Jiang X. Wang J. Li C. Wang L. Wang Z. Vapour pressure measurement for binary and ternary systems containing water methanol ethanol and an ionic liquid 1-ethyl-3ethylimidazolium diethylphosphate. J Chem Thermodyn 2007; 39: 841-46.

http://dx.doi.org/10.1016/j.jct.2006.11.013
[5] Wang J. Li Z. Li C, Wang Z. Density prediction of ionic liquids using a group contribution equation of state. Ind Eng Chem Res 2010; 49: 4420-25. http://dx.doi.org/10.1021/ie901590h

[6] Shen C. Li C. Li X. Lu Y. Muhammad Y. Estimation of densities of ionic liquids using Patel-Teja equation of state and critical parameters determined from group contribution method. Chem Eng Sci 2011; 66: 2690-98. http://dx.doi.org/10.1016/j.ces.2011.03.027

[7] Zhou Y. Robertson AJ. Hillhouse JH. Baumann D. Phosphonium and Imidazolium Salts and Methods of Their Preparation, WO 2004/016631 Al, 26 Feb. 2004.

[8] Liu G. Ma L. Liu J. Physical property for chemistry and chemical engineering, 1st ed. Chemical Industry Press, Beijing 2002.

[9] Wang J. Li C. Shen C. Wang Z. Towards understanding the electrostatic interaction on the density of ionic liquids. Fluid Phase Equilibria 2009; 279: 87-91. http://dx.doi.org/10.1016/j.fluid.2009.02.011

[10] Glasser L. Lattice and phase transition thermodynamics of ionic liquids. Thermochim Acta 2004; 421: 87-93. http://dx.doi.org/10.1016/j.tca.2004.03.015

[11] Huang ZQ. The Introduction of Electrolyte Solution Theory, divided ed. Science Press, Beijing 1983; p. 98.

[12] Tong J. Hong M. Guan W. Li JB. Yang JZ. Studies on the thermodynamic properties of new ionic liquids: 1-Methyl-3pentylimidazolium salts containing metal of group III. J Chem Thermodyn 2006; 38: 1416-21.

[13] Bhuiyan MMH. Uddin MH. Excess molar volumes and excess viscosities for mixtures of $\mathrm{N}, \mathrm{N}$-dimethylformamide with methanol, ethanol and 2-propanol at different temperatures. J Mol Liquids 2008; 138: 139-46. http://dx.doi.org/10.1016/j.molliq.2007.07.006

[14] Hofman T. Goldon A. Nevines A. Letcher TM. Densities, excess volumes, isobaric expansibity, and isothermal compressibility of the (1-ethyl-3-methylimidazolium ethylsulfate + methanol) system at temperatures (283.15 to 333.15) $\mathrm{K}$ and pressures from (0.1 to 35) $\mathrm{MPa}$. J Chem Thermodyn 2008; 40: 580-91. http://dx.doi.org/10.1016/i.jct.2007.11.011

[15] Wu Y, Bamgbade BA, Burgess WA, et al. Effect of Isomeric Structures of Branched Cyclic Hydrocarbons on Densities and Equation of State Predictions at Elevated Temperatures and Pressures. J Phy Chem B 2013; 117: 8821-30. http://dx.doi.org/10.1021/jp405795f

[16] Wu Y, Bamgbade BA, Baled $\mathrm{H}$, et al. Liquid Densities of Xylene Isomers and 2-Methylnaphthalene at Temperatures to $523 \mathrm{~K}$ and Pressures to $265 \mathrm{MPa}$ : Experimental Determination and Equation of State Modeling. Ind Eng Chemy Res 2013; 52: 11732-40. http://dx.doi.org/10.1021/ie400805y 